Check for updates

Cite this: RSC Adv., 2018, 8, 33666

Received 3rd August 2018

Accepted 11th September 2018

DOI: $10.1039 / \mathrm{c} 8 \mathrm{ra06555a}$

rsc.li/rsc-advances

\section{Nanowire network-based photodetectors with imaging performance for omnidirectional photodetecting through a wire-shaped structure $\uparrow$}

\author{
Shalong Wang, Yousheng Zou, * Qingsong Shan, Jie Xue, Yuhui Dong, Yu Gu \\ and Jizhong Song (DD*
}

\begin{abstract}
Wearable photodetectors (PDs) have attracted extensive attention from both scientific and industrial areas due to intrinsic detection abilities as well as promising applications in flexible, intelligent, and portable fields. However, most of the existing PDs have rigid planar or bulky structures which cannot fully meet the demands of these unique occasions. Here, we present a highly flexible, omnidirectional PD based on $\mathrm{ZnO}$ nanowire (NW) networks. ZnO NW network-based PDs exhibit the imageable level performance with an on/off ratio of about $10^{4}$. Importantly, a ZnO NW network can be assembled onto wire-shaped substrates to construct omnidirectional PDs. As a result, the wire-shaped PDs have excellent flexibility, a large light on/off ratio larger than $10^{3}$, and $360^{\circ}$ no blind angle detecting. Besides, they exhibit extraordinary stability against bending and irradiation. These results demonstrate a novel strategy for building wire-shaped optoelectronic devices through a NW network structure, which is highly promising for future smart and wearable applications.
\end{abstract}

\section{Introduction}

Wearable electronics and optoelectronics, which are also known as smart textiles or integration of electronic devices with textiles, have attracted extensive attention from both scientific and industrial areas. ${ }^{1-5}$ Among various smart devices, photodetectors (PDs) have a wide range of applications in missile detection, biological sensing, flame sensing, optical communications, astronomical studies, chemical analysis, medical diagnostics, digital imaging, environmental monitoring, remote control, and optoelectronic circuits, which have been extensively studied. ${ }^{\mathbf{6 - 1 4}}$ However, most existing PDs have planar or bulky structures, which cannot fully meet the urgent demands of portable, flexible, and wearable applications. ${ }^{\mathbf{1 5 , 1 6}}$ Compared with planar structures, wire-shaped or/and fibershaped photodetecting components, easily woven into textiles or other structures, have been proven to have a huge potential due to their high flexibility, knittability, small size and light weight. ${ }^{17-19}$

However, so far, the performances of wire-shaped PDs are still lower than their corresponding planar devices, because the existing wire-shaped PDs are mostly core-shell structures with

Institute of Optoelectronics \& Nanomaterials, MIIT Key Laboratory of Advanced Display Materials and Devices, School of Materials Science and Engineering, Nanjing University of Science \& Technology, Nanjing 210094, China. E-mail: songjizhong@njust.edu.cn; yshzou75@njust.edu.cn

$\uparrow$ Electronic supplementary information (ESI) available. See DOI: 10.1039/c8ra06555a an electrode inside the device and another electrode outside. Such a vertical structure will contribute to a high level of current in dark conditions, which more often than not cause low performance of the low on/off ratio. For example, $\mathrm{Ko}^{\mathbf{1 7}}$ et al. fabricated wire-shaped ultraviolet PDs based on a nanostructured zinc oxide $(\mathrm{ZnO}) /$ nickel oxide ( $\mathrm{NiO}$ ) coaxial $\mathrm{p}-\mathrm{n}$ heterojunction with a light on/off ratio of about 10. Chen $^{20}$ et al. reported a UV wire-PD with $\mathrm{ZnO}$ nanowires (NWs) grown on an oxidized Ni wire, and the light on/off ratio was about 4. A selfpowered fiber-shaped wearable omnidirectional ultraviolet (UV) PD with a light on/off ratio of 2 under zero bias was constructed by Dong and her co-authors. ${ }^{6}$ Therefore, it is desirable to design an all-purpose structure with excellent photodetecting performances for future wearable devices.

Various NW networks have been considered as a class of promising optoelectronic materials for flexible, stretchable, and wearable devices, owing to their superior elasticity for bending, stretching, twisting, and deformation into complex, non-planar shapes. ${ }^{21-24}$ In general, the strain applied by a mechanical stretching stress is accommodated by rotations and distortions of the NW network..$^{25-27}$ Thus, unconventional nanostructures, which are generated by spatial displacement between NWs with a high aspect ratio, can maintain their continuous network structures, which act as an electrical charge path, guaranteeing excellent carrier transporting properties under various deformations. Thus, there is a high potential to develop wire-shaped PDs based on a NWs network for wearable devices.

Here, we present omnidirectional PDs based on a ZnO NW network, which is promising for future wearable and smart 

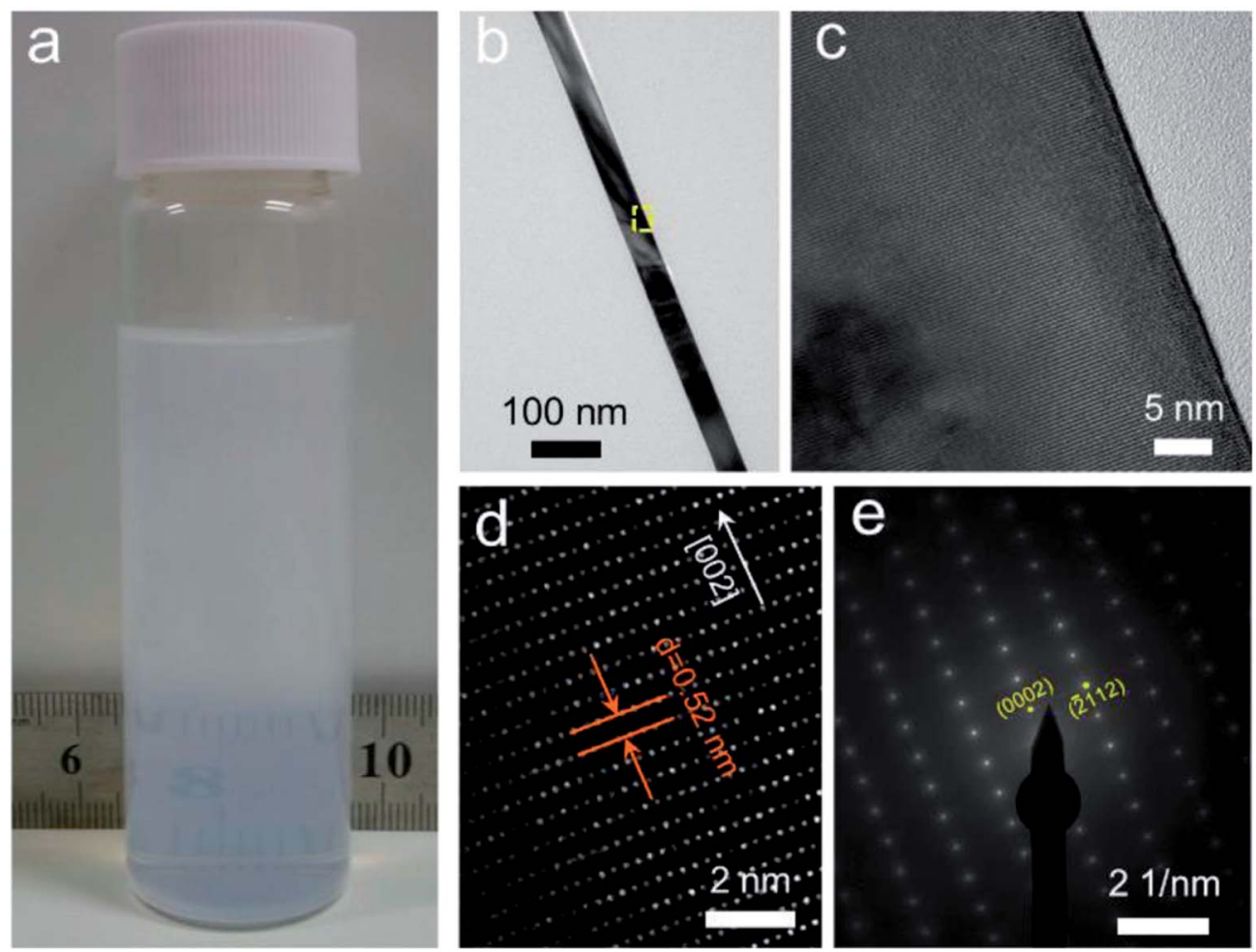

Fig. 1 (a) Photograph of ZnO NW inks re-dispersed in isopropanol. (b) TEM image of a ZnO NW with diameter of about $35 \mathrm{~nm}$. (c) Magnified TEM image of the surface of a ZnO NW. (d) High-resolution TEM image and (e) SAED pattern of ZnO NWs.
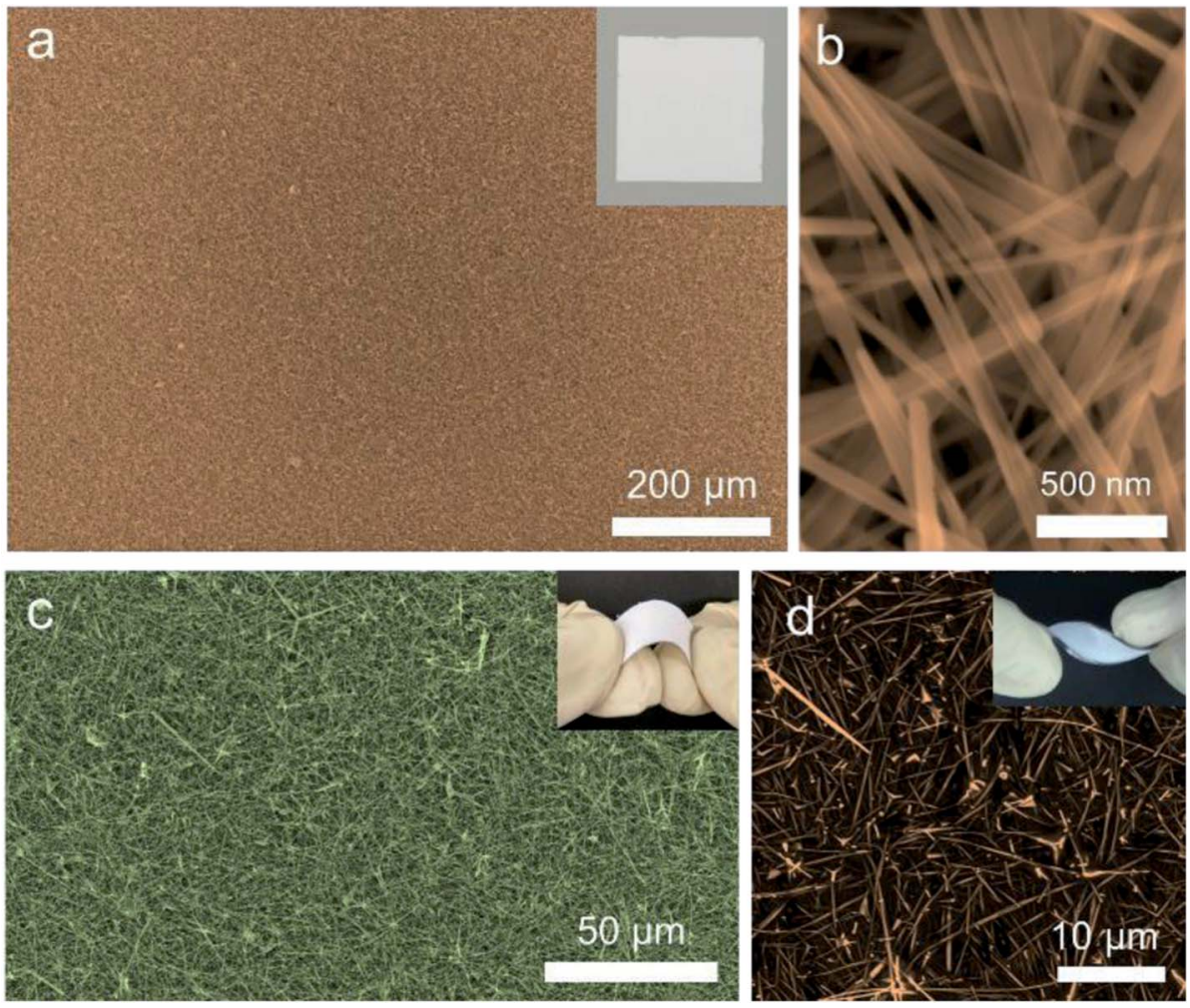

Fig. 2 ZnO NW networks on various substrates. (a) Large-area SEM images of the ZnO NW network film transferred on a glass substrates, inset: photograph of ZnO NW network film on a glass substrate. (b) High magnification SEM image of ZnO NWs on a glass substrate. SEM images of the ZnO NW network film transferred onto (c) PET and (d) PDMS substrates, insets: the corresponding photograph of ZnO NW network film on PET and PDMS substrates, respectively. 

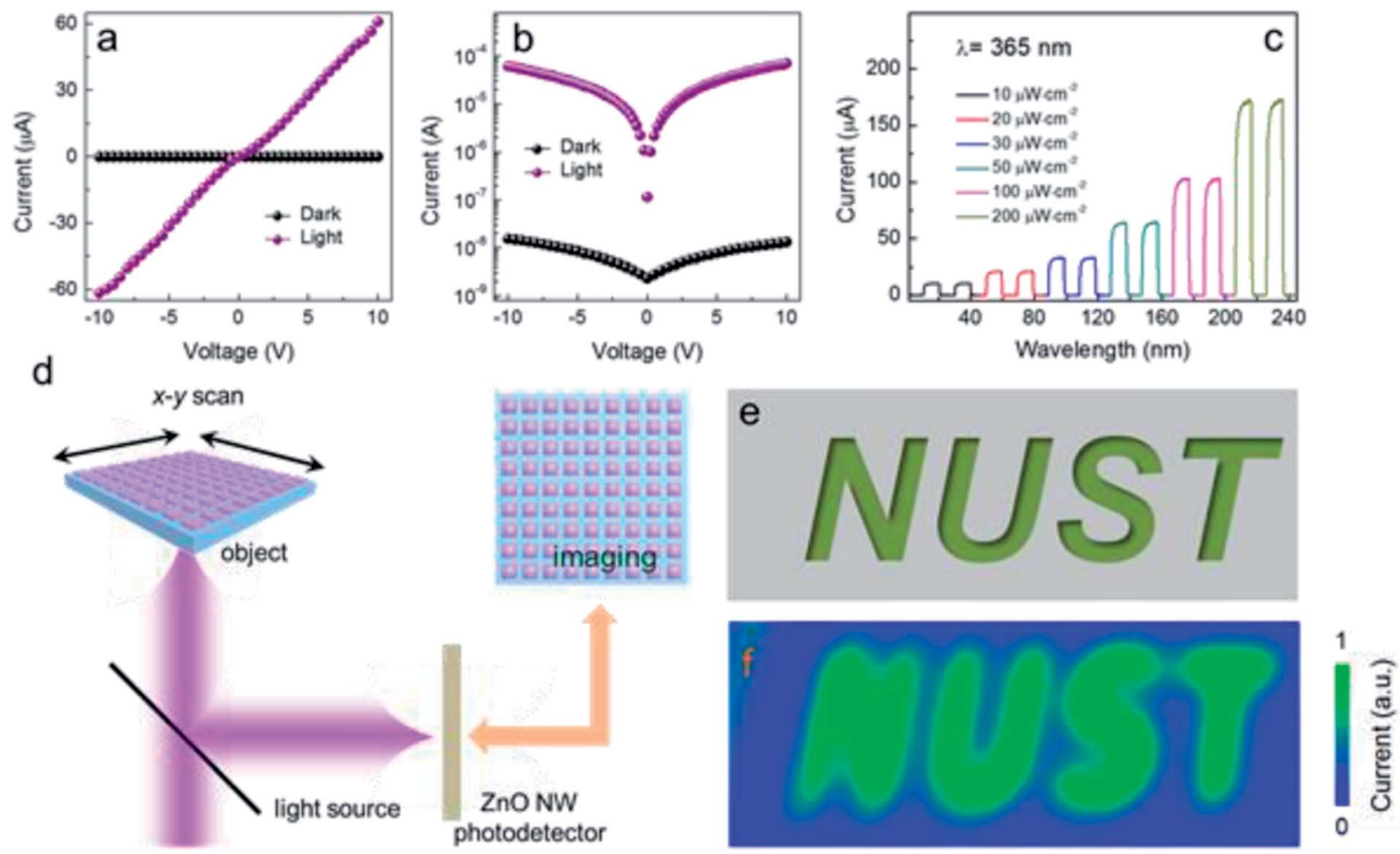

Fig. 3 Imageable photoelectric response characteristics of ZnO NW network. (a) I-V and (b) I-V logarithm characteristics of ZnO NW PD in the dark and under $365 \mathrm{~nm}\left(0.35 \mathrm{~mW} \mathrm{~cm}{ }^{-2}\right)$ illumination. (c) l-t curves at $5 \mathrm{~V}$ of ZnO NW PD under $365 \mathrm{~nm}$ with different light irradiation intensity. (d) Schematic of the PD imaging setup. (e) Object and (f) image acquired from the ZnO nanowire PD at $\lambda$ of $365 \mathrm{~nm}$.

optoelectronic devices. First, high quality ZnO NWs with large aspect ratios of $>100$ are readily dispersed in alcoholic solvents, e.g., isopropanol, serving as ink. The ink can be used to process highly uniform NWs network films for various optoelectronic devices. NW network-based PDs exhibit an imageable level performance with an on/off ratio of about $10^{4}$. Importantly, a ZnO NW network can be transferred onto wire-shaped substrates to construct omnidirectional PDs. As a result, the wire-shaped PDs have excellent flexibility ( $>500$ cycles), large light on/off ratio of $10^{3}$, and $360^{\circ}$ no blind angle detecting. These results demonstrate a novel strategy to build the wireshaped optoelectronic devices suitable for future smart and wearable applications.

\section{Experimental section}

\section{Synthetic procedures}

The as-obtained ZnO NWs were put in isopropanol and sonicated for $60 \mathrm{~s}$ to prepare a nanowire dispersion (typically with a concentration of $0.1 \mathrm{mg} \mathrm{mL}^{-1}$ ). Commercially available CNTs wire (diameter of $20-30 \mu \mathrm{m}$ and density of $0.3-0.5 \mathrm{~g} \mathrm{~cm}^{-3}$, from SCNC Company) was used as the electrode.

KEVLAR wire with diameter of $1 \mathrm{~mm}$ was dipped in PDMS gel and pre-cured at $60{ }^{\circ} \mathrm{C}$ for $1 \mathrm{~h}$. The PDMS (Sylgard 184, Dow Corning) gel was prepared from a mixture of a base and a curer (weight ratio $10: 1$ ). The KEVLAR wire was rotated on a PVDF filter membrane with the ZnO NWs. The sample was further twisted with CNT fiber wire to construct the PDs and then the devices were packaged with a thin layer of PDMS for testing.

\section{Materials characterization}

The morphologies of the NWs and devices were characterized using field-emission SEM (FE-SEM) (JSM 7600F). TEM images of ZnO NWs were measured with FEI Tecnai G20 on a Cu grid. The photoresponse behaviours of the devices were characterized using a Keithley 6487 analyzer with a portable UV LED as the light source $(365 \mathrm{~nm})$. Responsivities of the PDs were measured by a detector test system from ZOLIX INSTRUMENTS CO. Ltd with a Si PD as reference. The device was fixed on a home-made stretching stage to apply strain for flexible measurements. All measurements were performed at room temperature in ambient conditions.

\section{Results and discussion}

ZnO NW (purchased from Hebei Shaojing New Material Technology Co., Ltd.) can be readily dispersed in alcoholic solvents, e.g., isopropanol, serving as ink (Fig. 1a). The NW ink is compatible with several well-documented solution-processed thin-film deposition techniques, such as spray coating, scrape coating, and filtration. The ZnO NWs possess an average diameter of $\sim 35 \mathrm{~nm}$ (Fig. 1b) and an average length of more than $20 \mu \mathrm{m}$ (Fig. S1 $\dagger$ ). Such great length and large aspect ratio (>500) will favor their conductivity due to the resulting long and smooth transport channels for electrons. The microstructure of ZnO NWs was further confirmed by transmission electron microscopy (TEM) as shown in Fig. 1c-e. Fig. 1c clearly shows that the $35 \mathrm{~nm}$ NW has a very smooth and clean surface without any coating layer. The high resolution TEM (HRTEM) image in 

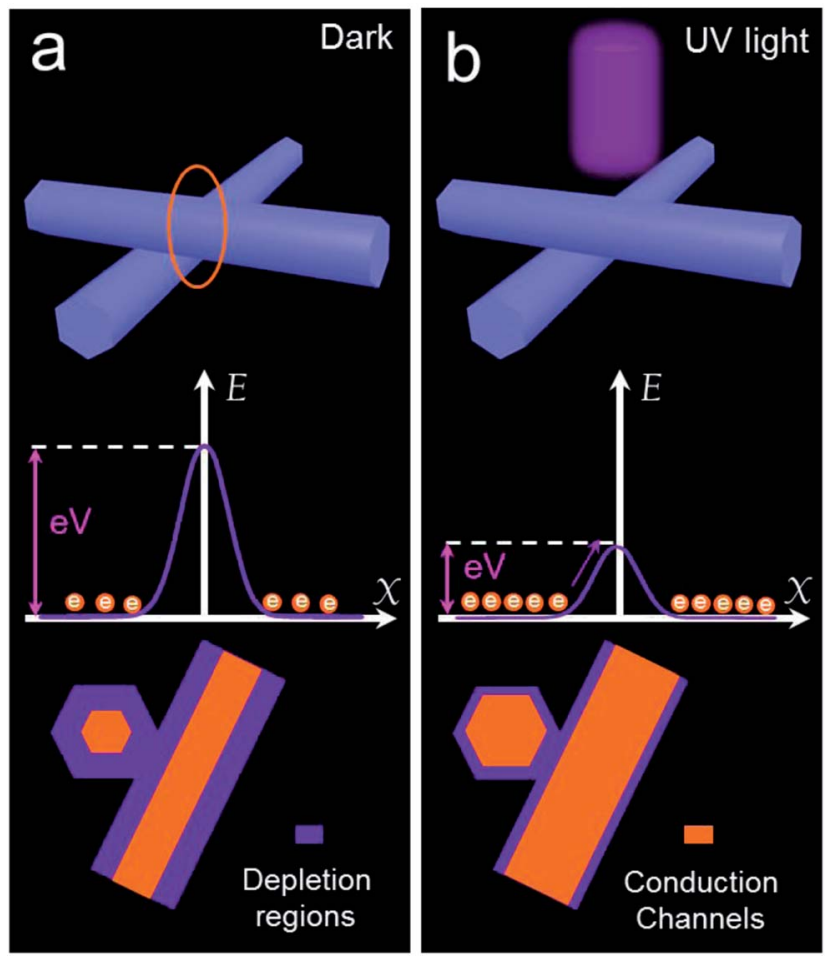

Fig. 4 Schematic representation of (top) a close binding NWs network bridge (a) without or (b) under UV irradiation and its corresponding (middle) energy band diagram in the radial direction and (bottom) cross-section structural models. The middle schematics in (b) present a reduced potential barrier at the boundary of two close binding NWs in cross-section that are irradiated with UV light (bottom) significantly increased channel.

Fig. 1d exhibits lattice fringes of $0.52 \mathrm{~nm}$, corresponding to the (001) plane normal to the growth direction, which is consistent with that of wurtzite $\mathrm{ZnO}$ crystal structure. ${ }^{28,29}$ Besides, the HRTEM image reveals a perfect lattice without any visible defects, which reflects the high crystallinity of ZnO NWs. The selected area electron diffraction (SAED) pattern shown in Fig. 1e also confirms that ZnO NWs have a good crystallinity, exhibiting a set of reflections corresponding to the [001] crystallographic direction of $\mathrm{ZnO}$ and being consistent with HRTEM. The high crystallinity ZnO NWs are very favorable for carrier transporting, which are highly promising in various optoelectronic devices.

As shown in Fig. 1a, upon sonication for $1 \mathrm{~min}$, the ZnO NWs formed a dispersion in isopropanol. The NW inks were filtered with a polyvinylidene fluoride (PVDF) membrane to form a thin and uniform film, which then could be easily transferred onto various substrates. Fig. 2a shows the large-area $\mathrm{ZnO} \mathrm{NW}$ network transferred onto glass substrates characterized by SEM images. The ZnO NWs lay on the substrate due to their unique 1D geometries, and can be assembled into dense, crack-free, and smooth films observed by the photograph (inset in Fig. 2a). The high magnification SEM image shown in Fig. 2b demonstrates that ZnO NWs are intertwined together to form a perfect network structure. As described in the introduction, such network structure, which is very favorable for adapting to the deformations under applied stress, could maintain high flexibility. Accordingly, the flexible ZnO NWs network transferred onto flexible polyethylene terephthalate (PET) and stretchable polydimethylsiloxane (PDMS) substrates are shown in Fig. 2c and d. Especially, after bending and recovering for about 500 times the NWs on the PDMS substrates still retained their integrity. This is ascribed to the NWs being partially embedded in PDMS polymer, which anchored the NW network and further preserved the integrity of junctions among the NWs.

The photoelectric response characteristics of a $\mathrm{ZnO} \mathrm{NW}$ network are presented in Fig. 3. The device configuration of a ZnO NW network PD is schematically demonstrated in Fig. $\mathrm{S} 2 ; \dagger \mathrm{Au}$ interdigital electrodes were deposited on $\mathrm{ZnO}$ network substrates. Fig. $3 \mathrm{a}$ and $\mathrm{b}$ show the $I-V$ and $I-V$ logarithm characteristics of a ZnO NW network PD in the dark and under $365 \mathrm{~nm}\left(0.35 \mathrm{~mW} \mathrm{~cm}^{-2}\right)$ illumination, respectively. The linear $I-V$ relationship with forward and reverse bias measured in the dark clearly shows an approximate ohmic contact between the ZnO NW network and electrodes. ${ }^{30,31}$ The NW network exhibits high photoresponse. For example, the dark
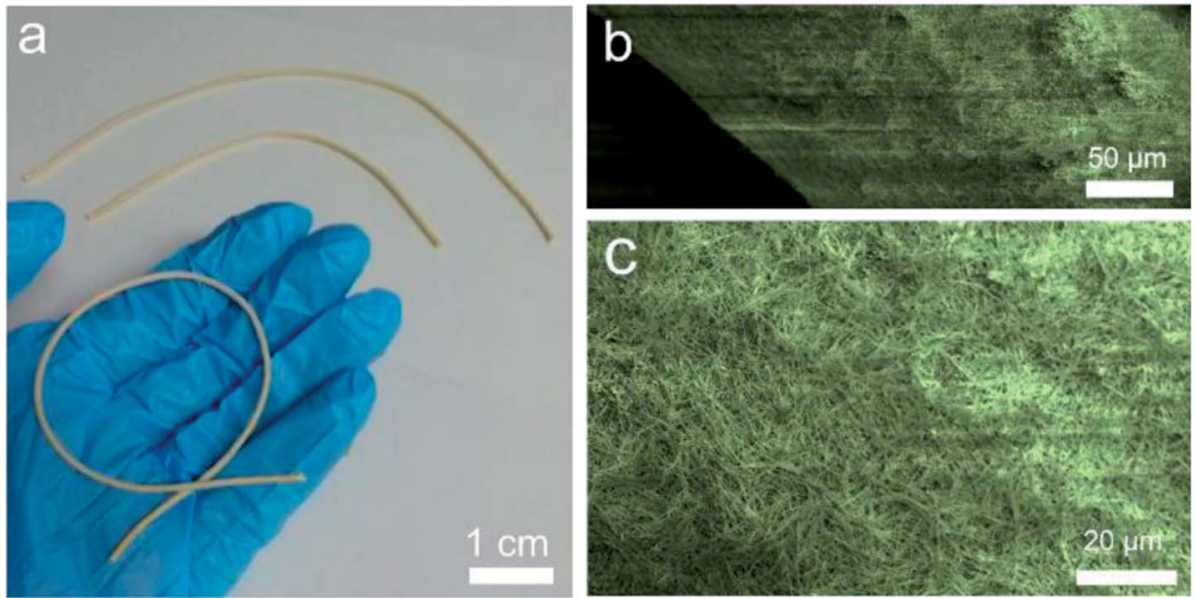

Fig. 5 (a) Photograph of a ZnO NW network coated on wire-shaped KEVLAR. SEM image (b) and high magnification SEM image (c) of the ZnO NW network film coated on KEVLAR substrates. 

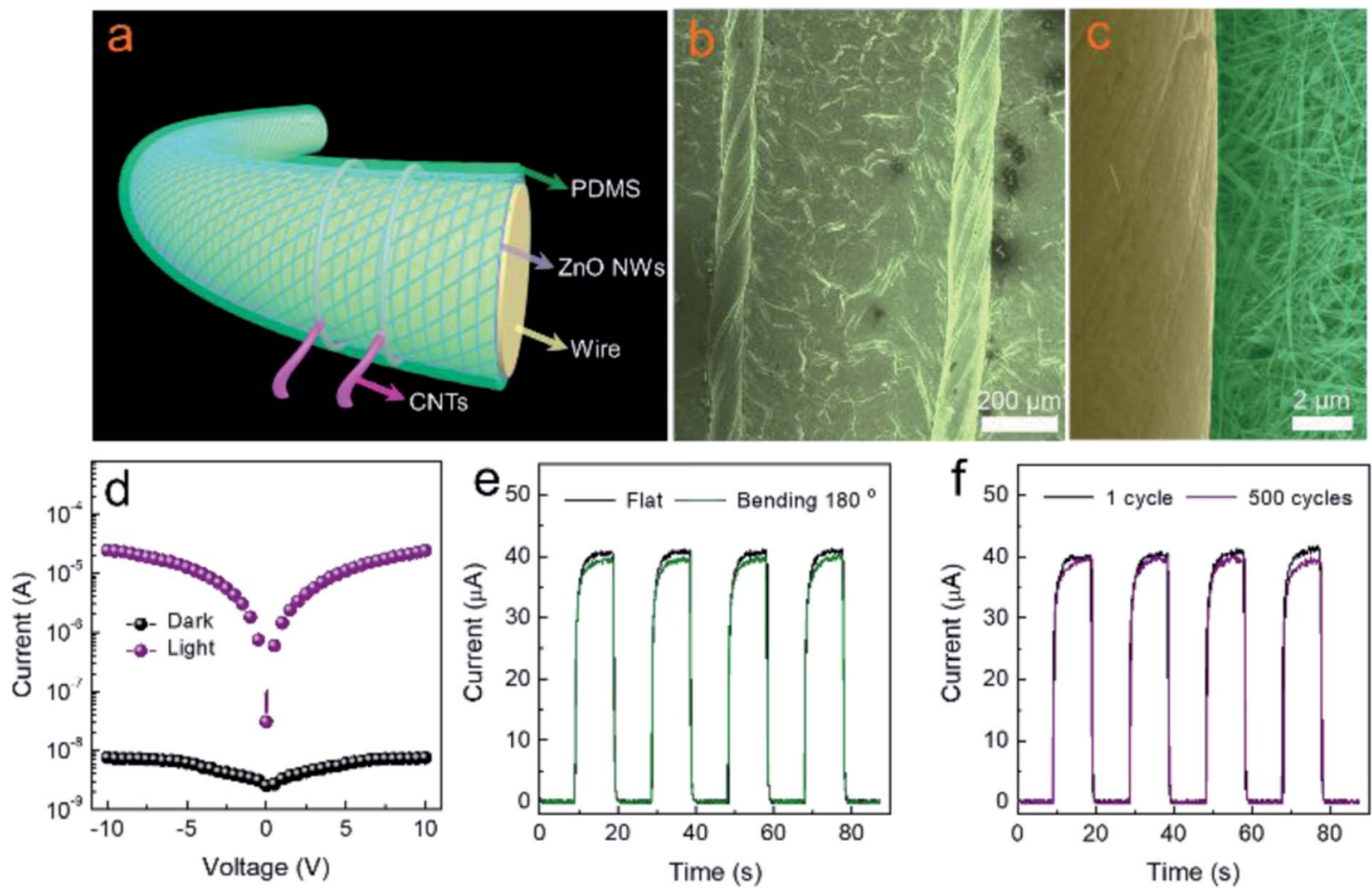

Fig. 6 (a) Device structure of wire-shaped ZnO PD. (b) SEM image and (c) high magnification SEM image of wire-shaped ZnO PD. (d) I-V logarithm characteristics of ZnO wire-shaped NW PD in the dark and under $365 \mathrm{~nm}\left(0.35 \mathrm{~mW} \mathrm{~cm}^{-2}\right)$ illumination. (e) $I-t$ curves at $2 \mathrm{~V}$ of ZnO NW PD under $365 \mathrm{~nm}$ after bending and recovering cycles. (f) I-t curves at $2 \mathrm{~V}$ of ZnO NW PD under $365 \mathrm{~nm}$ with different cycle times.

current and photocurrent are $7.73 \mathrm{nA}$, and $30 \mu \mathrm{A}$ at $5 \mathrm{~V}$ applied bias, respectively whereby the resulting on/off current ratio was calculated to be $3.9 \times 10^{3}$ at $5 \mathrm{~V}$.

To illustrate the light-switching characteristic, the photocurrent-time response measured in the dark and under illumination was controlled by a light shutter. As shown in Fig. S3, $\uparrow$ when the UV light was turned on, the photocurrent sharply increased under the applied bias voltage. Meanwhile, the device immediately decayed to a dark current as soon as the UV light was turned off. The device exhibited stable and reproducible responses to on-off cycles. The current-time $(I-t)$ curves at $2 \mathrm{~V}$

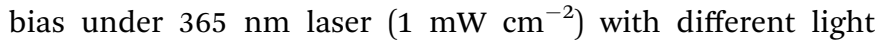
irradiation intensity are presented in Fig. 3c. As shown in this picture, under illumination the device produced a photocurrent which steadily increased with increasing light power from 10 $\mu \mathrm{W} \mathrm{cm}{ }^{-2}$ to $200 \mu \mathrm{W} \mathrm{cm}{ }^{-2}$. Especially, the current measured at $2 \mathrm{~V}$ reached a maximum value of $64 \mu \mathrm{A}$ with a light intensity of $50 \mu \mathrm{W} \mathrm{cm}{ }^{-2}$, which is about 3 orders of magnitude compared with that under dark conditions.

Furthermore, the spectral responsivity of the device considered as a function of excitation wavelengths was measured. The responsivity $R_{\lambda}$ was calculated according to the following equation: $:^{32}$

$$
R_{\lambda}=\frac{I_{\mathrm{ph}}}{P_{0} S}
$$

where $I_{\mathrm{ph}}$ is the difference between the light current and dark current $\left(I_{\mathrm{ph}}=I_{\text {light }}-I_{\text {dark }}\right), P_{0}$ is the irradiance power density on the NTs, and $S$ is the effective illuminated area. The responsivity and external quantum efficiency (EQE) of the PD were obtained by using a Si PD as a reference (see Fig. S4 $\dagger$ ). The responsivity decreased sharply after $365 \mathrm{~nm}$, showing its spectral cutoff characteristic. The cutoff wavelength is estimated to be about $380 \mathrm{~nm}$, which agrees quite well with their energy bandgap. The maximum value of the responsivity and EQE located at a wavelength of $360 \mathrm{~nm}$ are $1.43 \mathrm{~A} \mathrm{~W}^{-1}$ and $467 \%$, respectively.

The detectivity $\left(D^{*}\right)$, demonstrated the ability of the photodetector for detecting a weak signal, also a critical parameter, which is calculated by the following equation:

$$
D^{*}=\frac{R_{\lambda} A^{1 / 2}}{\left(2 e I_{\mathrm{dark}}\right)^{1 / 2}}
$$

where $A$ is the working area, $e$ is the electron charge and $I_{\text {dark }}$ refers to the dark current. Therefore, the detectivity can be calculated to be $7.22 \times 10^{11}$ Jones.

To further demonstrate the high photoresponse properties, a distinct imaging picture with letters of NUST (Nanjing University of Science and Technology) is demonstrated in Fig. 3d-f. The photocurrent microscopy system was constructed as shown in Fig. 3d (complete imaging setup is shown in Fig. S5 $\dagger$ ). Through a current pre-amplifier and lock-in amplifier, signal photocurrents are extracted from the NW network-based device to form images. ${ }^{33-35}$ Fig. 3e shows a metallic object (NUST) with a line width of $5 \mu \mathrm{m}$ patterned. The metallic objects 

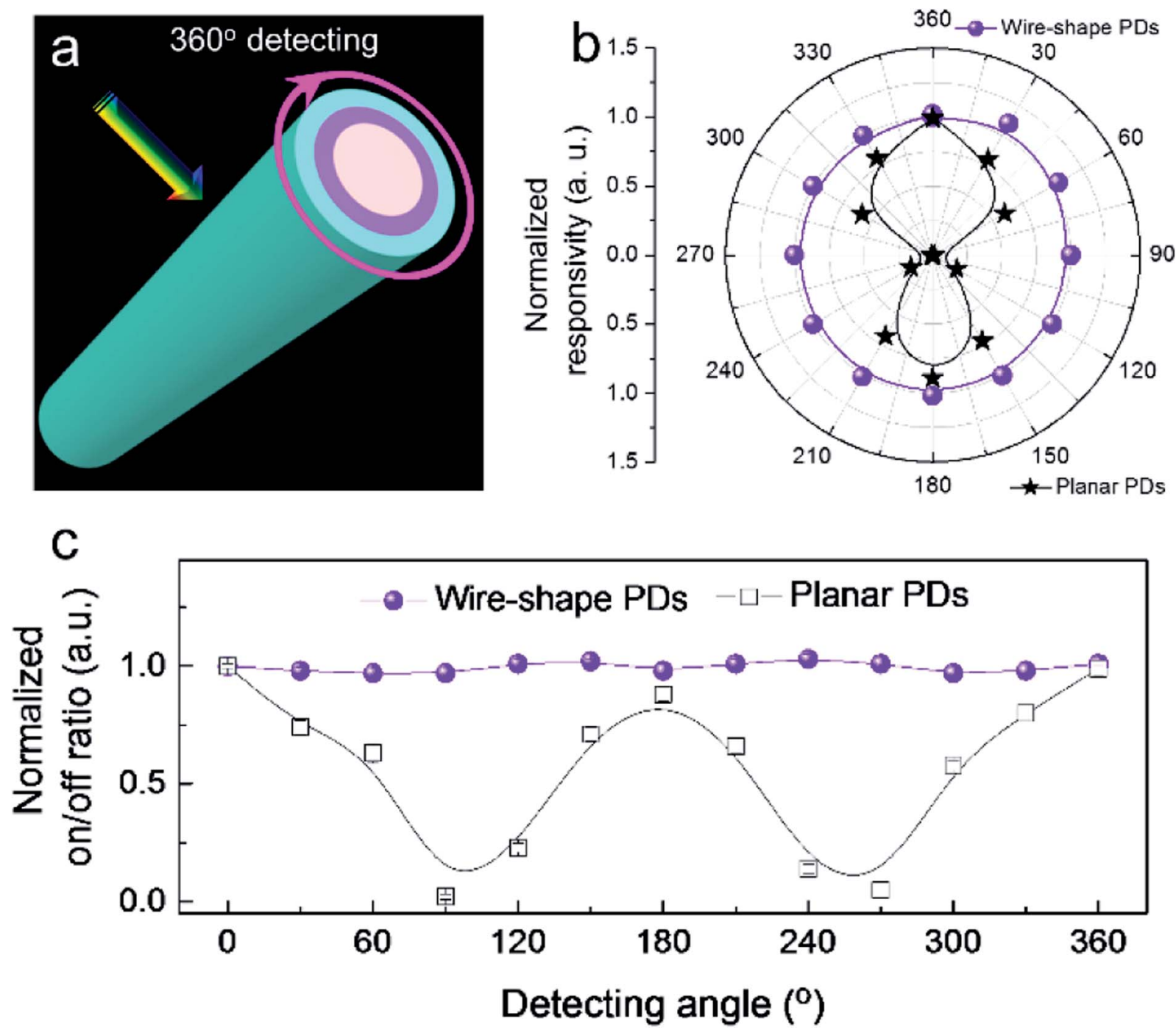

Fig. 7 (a) Schematic of the $360^{\circ}$ omnidirectional PDs. (b) Dependence of normalized responsibility for wire-shaped and planar PD at different detecting angles. (c) Dependence of on/off ratio and detecting angle for wire-shaped and planar PDs.

can be any other patterns with any shape or size. The corresponding images were acquired with the NW network-based detector by applying a focused light spot (365 nm) scanning across the patterns. Simultaneously, signal photocurrents from a pattern edge cross section in the images were extracted (see Fig. 3f). The location of photocurrent and background appeared in sharp contrast with the clear imaging letters on the picture. Meanwhile, the green color of the letter indirectly reflects the highly uniform feature of a NW network.

Such high performances (low dark current, high photocurrent, high responsivity, etc.) are attributed to a special carrier transport mechanism. Consequently, the generation of carriers and their transport in a NW network photodetector are depicted in Fig. 4. Usually, higher dark current is presented in a $\mathrm{ZnO}$ film photodetector, where the carriers have a smoother channel than in a NW network-based photodetector. In general, on a NW surface, oxygen molecules capture free electrons through $\left[\mathrm{O}_{2}(\mathrm{~g})\right.$ $+\mathrm{e}^{-} \rightarrow \mathrm{O}_{2}{ }^{-}$(ad)], leading to a low conductivity surface depletion layer under dark conditions. Carrier density in the NWs increased considerably under UV light ( $h v>E_{\mathrm{g} \text { ZnO}}$ ), by electrons directly transported from the valence band to the conduction band. Adsorbed oxygen ions were discharged by the photogenerated holes through $\left[\mathrm{O}_{2}{ }^{-}(\mathrm{ad})+\mathrm{h}^{+} \rightarrow \mathrm{O}_{2}(\mathrm{~g})\right]$, reducing the thickness of the depletion barrier. ${ }^{36,37}$ Thus, the increase in carrier density and oxygen desorption contributes to the conductance increase in the NWs. Besides, the interaction between NWs also needs to be considered in NW network-based devices because the resistance of two crossed NWs was dominated by the NW-NW junction barrier and the resistances of the NWs themselves. In our ZnO NW network PDs, the electrons have to overcome the NWs-NWs junction barrier when tunneling from one NWs to another. ${ }^{38,39}$ The electron-transfer barrier originates from the surface depletion layers. As discussed in single NWs, the depletion layer can be narrowed by UV illumination due to increased carrier density, which is equivalent to a lowering of the effective barrier height. ${ }^{39,40}$ It is 
thus easier for electrons to go through the network upon UV illumination, therefore resulting in the increase of current.

Importantly, the NW network films can be integrated with wire-shaped substrates suitable for future wearable and smart devices, which can release part of bending stress and are suitable for wearable optoelectronic fields. A photograph of a $\mathrm{ZnO}$ NW network coated on KEVLAR exhibiting high flexibility is shown in Fig. 5a. The wire-shaped PD was fabricated by rolling a tensioning KEVLAR wire on the NW network substrates, which is illustrated in Fig. S6. $\dagger$ The film microstructure on the KEVLAR wire surface was characterized by SEM images (Fig. 5b and c). Similar to NW network films on planner substrates, the networks on wire-shaped substrates also exhibit high quality, crack-free, and smooth surfaces.

The NW networks on KEVLAR can be assembled into wireshaped PDs by twining the CNT electrodes. The structure of a wire-shaped PD is demonstrated in Fig. 6a. The KEVLAR wire substrate is coated by ZnO NWs with CNTs as the electrode. The device's structure was confirmed by the SEM images shown in Fig. $6 \mathrm{~b}$ and $\mathrm{c}$. The contact between CNT and the ZnO NW network is good due to the excellent flexibility of CNT, which enables charge collecting from the NWs active layers.

The wire-shaped PDs also exhibit high photoresponse features compared with those of a corresponding planner structure. As shown in Fig. 6d, the dark current and photocurrent for wire-shaped PDs are $4.7 \mathrm{nA}$ and $5.71 \mu \mathrm{A}$ at $2 \mathrm{~V}$ applied bias, respectively. The on/off current ratio was calculated to be $1.2 \times 10^{3}$ at $2 \mathrm{~V}$. Meanwhile, the wire-shaped PDs exhibited high flexibility. The photoresponse behavior of the device under different bending situations was characterized by measuring the current as a function of time when a $365 \mathrm{~nm}$ UV light was periodically turned on and off (Fig. S7 $\dagger$ ). The current-time $(I-t)$ curves at $2 \mathrm{~V}$ bias under $365 \mathrm{~nm}$ laser $\left(1 \mathrm{~mW} \mathrm{~cm}^{-2}\right)$ under a flat conformation and bending $180^{\circ}$ are respectively presented in Fig. 6e, with almost no change in the photocurrent value, which illustrates extraordinarily flexibility of our wire-shaped PDs. Additionally, after 500 cycles of bending tests, the $I-t$ curves still remained at the same level (Fig. 6f). These good mechanical properties probably resulted from the network structure of $\mathrm{ZnO}$ NWs. SEM images of wire-shaped ZnO PD after bending for 500 times are illustrated in Fig. S8, $\uparrow$ showing compactness and interconnecting NWs in the PD after 500 cycles without severe damage.

Importantly, the wire-shaped structure possesses excellent features such as less limitation of incident light angle, wider range of the substrate materials and wearability, and especially it can absorb light in incident directions and greatly diversified applications of PDs. The relationship between normalized responsivity and detecting angle is shown in Fig. 7. Obviously, the responsiveness of planar PDs decreases under side irradiation while the wire-shaped $360^{\circ}$ PDs are capable of detecting incident directions and show no blind spots when compared with rigid planar structure PDs (Fig. 7b). Due to their unique structure, the performances (e.g., on/off ratio) of the $360^{\circ}$ PDs remained almost unchanged under different bending angles with the same light intensity (Fig. 7c). Therefore, they can be efficiently used for wearable optoelectronic fields. Besides, a wire-shaped PD is also capable of long-time operating as the stability of $I_{\text {light }} v s$. time demonstrated in Fig. S9† in which the photocurrent showed no decrease after 12 hours irradiation.

\section{Conclusions}

In conclusion, we demonstrated highly flexible, omnidirectional PDs based on a ZnO NW network. PDs based on this NW network exhibit excellent performance with high-resolution imaging and an on/off ratio as high as about $10^{4}$. Importantly, ZnO NW networks can be transferred onto wire-shaped substrates to construct omnidirectional PDs. As a result, wireshaped PDs have excellent flexibility and a large light on/off ratio of $10^{3}$. Besides, the omnidirectional PDs exhibit extraordinary stability against bending and irradiation. These results demonstrate a novel strategy for building wire-shaped optoelectronic devices through a NW network, which has high potential for future smart and wearable applications.

\section{Conflicts of interest}

There are no conflicts to declare.

\section{Acknowledgements}

We acknowledge Hehai Fang and Professor Weida $\mathrm{Hu}$ in Shanghai Institute of Technical Physics, Chinese Academy of Sciences for the support of photodetector imaging. This work was financially supported by the National Key Research and Development Program of China (2017YFA0305500, 2016YFB0401701), NSFC (61604074, 51572128, 51672132, 61725402), the Natural Science Foundation of Jiangsu Province (BK20160827, BK20180020), China Postdoctoral Science Foundation (2016M590455), the Fundamental Research Funds for the Central Universities (No. 30917011202, 30915012205, and 30916015106), and PAPD of Jiangsu Higher Education Institutions.

\section{Notes and references}

1 J. Zhong, Y. Zhang, Q. Zhong, Q. Hu, B. Hu, Z. L. Wang and J. Zhou, ACS Nano, 2014, 8, 6273-6280.

2 N. Yu, H. Yin, W. Zhang, Y. Liu, Z. Tang and M. Q. Zhu, Adv. Energy Mater., 2016, 6, 1501458.

3 Z. Bao and X. Chen, Adv. Mater., 2016, 28, 4177-4179.

4 J. Xue, J. Song, Y. Dong, L. Xu, J. Li and H. Zeng, Sci. Bull., 2017, 62, 143-156.

5 Z. Zhang, K. Guo, Y. Li, X. Li, G. Guan, H. Li, Y. Luo, F. Zhao, Q. Zhang and B. Wei, Nat. Photonics, 2015, 9, 233.

6 Y. Dong, Y. Zou, J. Song, Z. Zhu, J. Li and H. Zeng, Nano Energy, 2016, 30, 173-179.

7 N. Nasiri, R. Bo, L. Fu and A. Tricoli, Nanoscale, 2017, 9, 2059-2067.

8 Z. Zhu, Y. Gu, S. Wang, Y. Zou and H. Zeng, Adv. Electron. Mater., 2017, 3, 1700281.

9 M. Qiu, P. Sun, Y. Liu, Q. Huang, C. Zhao, Z. Li and W. Mai, Adv. Mater. Technol., 2018, 3, 1700288. 
10 S. Pyo, W. Kim, H. I. Jung, J. Choi and J. Kim, Small, 2017, 13, 1700918.

11 Y. Zhang, X. Q. Yan, Y. Yang, Y. H. Huang, Q. L. Liao and J. J. Qi, Adv. Mater., 2012, 24, 4647-4655.

12 P. Lin, X. Chen, X. Q. Yan, Z. Zhang, H. G. Yuan, P. F. Li, Y. G. Zhao and Y. Zhang, Nano Res., 2014, 7, 860-868.

13 S. Liu, Q. L. Liao, Z. Zhang, X. K. Zhang, S. N. Lu, L. X. Zhou, M. Y. Hong, Z. Kang and Y. Zhang, Nano Res., 2017, 10, 34763485.

14 Z. Zhang, Q. L. Liao, Y. H. Yu, X. D. Wang and Y. Zhang, Nano Energy, 2014, 9, 237-244.

15 T. Gao, Q. Zhang, J. Chen, X. Xiong and T. Zhai, Adv. Opt. Mater., 2017, 5, 1700206.

16 N. Nasiri, R. Bo, F. Wang, L. Fu and A. Tricoli, Adv. Mater., 2015, 27, 4336-4343.

17 Y. H. Ko, G. Nagaraju and J. S. Yu, Nanoscale, 2015, 7, 27352742.

18 Z. Zhu, D. Ju, Y. Zou, Y. Dong, L. Luo, T. Zhang, D. Shan and H. Zeng, ACS Appl. Mater. Interfaces, 2017, 9, 12092-12099.

19 X. Wang, K. Jiang and G. Shen, Mater. Today, 2015, 18, 265272.

20 J. Chen, L. Ding, X. Zhang, L. Chu, N. Liu and Y. Gao, Opt. Express, 2014, 22, 3661-3668.

21 H. Moon, H. Lee, J. Kwon, Y. D. Suh, D. K. Kim, I. Ha, J. Yeo, S. Hong and S. H. Ko, Sci. Rep., 2017, 7, 41981.

22 H.-G. Im, S.-H. Jung, J. Jin, D. Lee, J. Lee, D. Lee, J.-Y. Lee, I.-D. Kim and B.-S. Bae, ACS Nano, 2014, 8, 10973-10979.

23 J. Song, J. Li, J. Xu and H. Zeng, Nano Lett., 2014, 14, 62986305.

24 J. Song and H. Zeng, Angew. Chem., Int. Ed., 2015, 54, 97609774.

25 Y. Won, A. Kim, W. Yang, S. Jeong and J. Moon, NPG Asia Mater., 2014, 6, e132.
26 T. Cheng, Y. Zhang, W. Y. Lai and W. Huang, Adv. Mater., 2015, 27, 3349-3376.

27 K. K. Kim, S. Hong, H. M. Cho, J. Lee, Y. D. Suh, J. Ham and S. H. Ko, Nano Lett., 2015, 15, 5240-5247.

28 J. Song, S. A. Kulinich, J. Yan, Z. Li, J. He, C. Kan and H. Zeng, Adv. Mater., 2013, 25, 5750-5755.

29 J. Song, S. A. Kulinich, J. Li, Y. Liu and H. Zeng, Angew. Chem., 2015, 127, 472-476.

30 G. Li, J. Zhang, G. Chen, H. Ye, X. Duan and X. Hou, SolidState Electron., 2016, 123, 33-37.

31 J. Song, L. Xu, J. Li, J. Xue, Y. Dong, X. Li and H. Zeng, Adv. Mater., 2016, 28, 4861-4869.

32 L. Li, P. Wu, X. Fang, T. Zhai, L. Dai, M. Liao, Y. Koide, H. Wang, Y. Bando and D. Golberg, Adv. Mater., 2010, 22, 3161-3165.

33 N. Guo, W. Hu, T. Jiang, F. Gong, W. Luo, W. Qiu, P. Wang, L. Liu, S. Wu and L. Liao, Nanoscale, 2016, 8, 16065-16072.

34 M. Engel, M. Steiner and P. Avouris, Nano Lett., 2014, 14, 6414-6417.

35 D. K. Hwang, Y. T. Lee, H. S. Lee, Y. J. Lee, S. H. Shokouh, J.-h. Kyhm, J. Lee, H. H. Kim, T.-H. Yoo and S. H. Nam, NPG Asia Mater., 2016, 8, e233.

36 C. Y. Yan, N. Singh and P. S. Lee, Appl. Phys. Lett., 2010, 96, 044507.

37 M. R. Alenezi, S. J. Henley and S. Silva, Sci. Rep., 2015, 5, 8516.

38 J. Wang, C. Yan, M.-F. Lin, K. Tsukagoshi and P. S. Lee, J. Mater. Chem. C, 2015, 3, 596-600.

39 Z. Zhan, L. Xu, J. An, H. Du, Z. Weng and W. Lu, Adv. Eng. Mater., 2017, 19, 1700101.

40 G. Cheng, X. H. Wu, B. Liu, B. Li, X. T. Zhang and Z. L. Du, Appl. Phys. Lett., 2011, 99, 203105. 